Revue d'histoire de l'Amérique française

Q REVUE D.HISTOIRE DE L'AMÉRIQUE FRANÇAISE

\title{
Les origines de la famille Le Moine
}

\section{Joseph Le Ber}

Volume 1, numéro 1, juin 1947

URI : https://id.erudit.org/iderudit/801349ar

DOI : https://doi.org/10.7202/801349ar

Aller au sommaire du numéro

Éditeur(s)

Institut d'histoire de l'Amérique française

ISSN

0035-2357 (imprimé)

1492-1383 (numérique)

Découvrir la revue

Citer cet article

Le Ber, J. (1947). Les origines de la famille Le Moine. Revue d'histoire de l'Amérique française, 1(1), 101-107. https://doi.org/10.7202/801349ar d'utilisation que vous pouvez consulter en ligne.

https://apropos.erudit.org/fr/usagers/politique-dutilisation/ 


\section{LES ORIGINES DE LA FAMILLE LE MOINE}

Les origines de la famille LE MOINE, une des plus illustres du CANADA, sont presque totalement ignorées; on sait seulement que le fondateur de cette lignée remarquable fut Charles LE MOINE. C'était le fils d'un hôtelier Dieppois, d'aucuns disent même d'un cabaretier; il avait 15 ans lorsqu'en 1641 son oncle Adrien DUCHESNE le fit venir au CANADA où il ne fut d'abord que simple soldat et interprète, mais ce Dieppois et ses onze fils étaient promis à de glorieuses destinées et ils devaient rendre célèbres dans toute l'Amérique du Nord des noms de chez nous, les noms de LONGUEUIL et d'IBERVILLE qu'ils y portèrent.

Notre but, dans cette étude, est de donner quelques renseignements généalogiques afin de faire connaître, dans une trop faible mesure, hélas, ce qu'étaient les LE MOINE et les DUCHESNE. Chemin faisant nous tâcherons d'identifier cette hostellerie ou cabaret d'où partit Charles LE MOINE et nous comprendrons mieux aussi pourquoi, devenu noble, il donna à ses fiefs les noms de LONGUEUIL et d'IBERVILLE, des noms de la Région Dieppoise.

\section{LA FAMILLE DUCHESNE}

C'est, dit-on, à l'appel de son oncle, Adrien DUCHESNE, que Charles LE MOINE partit pour le Canada. Voyons ce qu'était la famille DUCHESNE.

Adrien DUCHESNE, chirurgien à QUEBEC puis aux TROIS RIVIERES était le frère de Judith DUCHESNE, mère de Charles LE MOINE. D'où venait-il? Quelle était cette famille DUCHESNE? C'est ce que nous allons essayer d'abord d'éclaircir.

La famille DUCHESNE est, semble-t-il, originaire de LONGUEUIL ${ }^{1}$, village de 500 Habitants, à $12 \mathrm{Kms}$ environ de DIEPPE.

1. Canton d'OFFrANVILLE. 
Adrien DUCHESNE et son père y possédaient quelques biens et y récidèrent; peut-être y sont-ils nés l'un et l'autre, mais nous ne pouvons l'affirmer.

Vers 1560 un Nicolas DUCHESNE était fermier de la pêcherie de LONGUEUIL ${ }^{1}$. En 1595, "honnête femme Catherine LFFAUDEUR, veuve de défunt Maitre ETIENNE DUCHESNE " habitait également LONGUEUIL, ainsi qu'Abraham DUCHESNE et Thomas son frère; nous avons même, quant à ABRAHAM, une précision, il demeurait au hameau de BLAINVILLE?

Abraham DUCHESNE n'était-il pas un oncle d'Adrien DU. CHESNE? Peut-être, en tout cas ils étaient parents probablement car le 18 Novembre 1598 Pierre DUCHESNE, le père d'Adrien, en qualité d'héritier de "défunte Jeanne CAMPIGNY sa mère ", rendait aveu au duc de LONGUEVILLE pour une petite pièce de terre située au terroir de BLAINVILLE, et 20 ans plus tard, en 1618, le même Pierre DUCHESNE ratifie "l'aveu pour lui présenté par Abraham DUCHESNE d'une pièce de terre en labour assise au dit LONGUEUIL, terroir de BLAINVILLE ${ }^{3}{ }^{3}$.

Nous pouvons donc dès maintenant commencer avec certitude notre généalogie des DUCHESNE. Elle débute comme suit:

N. DUCHESNE

Jeanne CAMPIGNY

décédée avant le 18 Novembre 1598

\section{PIERRE DUCHESNE}

10) PIERRE DUCHESNE :

A la fin du XVIe Siècle, Maître Pierre DUCHESNE était contrôleur pour le Roi des aides et tailles en l'élection d'ARQUES; il demeurait en la paroisse de LONGUEUIL, non pas au hameau de BLAINVILLE, mais en celui de TOUS LES MESNILS et il se qualifiait déjà Sieur d'IBERVILLE ${ }^{4}$.

1. Archives de la Seine-Inférieure C. 2.813.

2. T.B. (c'est-à-dire Tabellionage de BACQUEVILLE) - 26 Juin et 11 Décembre 1595. Nous devons à l'obligeance de Maître JEAN, Notaire à BACQUEVILLE EN CAUX d'avoir pu consulter ces vieux registres. Qu'il en soit ici remercié. P. 331 .

3. Bibliothèque de Dieppe-Inventaire du duché de LONGUEVILLE (en 1694)

4. T.B. 11 Juillet 1595. 
Voici done que nous voyons pour la première fois apparaître dans la famille DUCHESNE ce nom qu'un ILE MOINE rendra célèbre. Où se trouvait ce fief?

Dans la région de DIEPPE il y en avait deux qui portaient ce nom qu'on écrivait tantôt YBERVILLE avec un y, tantôt IBERVILLE avec un I; l'un situé en la paroisse du THIL ${ }^{1}$ et qui maintenant désigne une ferme importante; l'autre totalement ignoré de nos jours, dans la paroisse de TOURVILLE sur ARQUES, à 3 Kilomètres environ du premier ${ }^{2}$. Longtemps nous avons cru qu'il s'agissait du fief situé au THIL jusqu'au jour où un acte de tabellionage nous apprit que le fief d'IBERVILLE appartenant à Pierre DUCHESNE se trouvait sur la paroisse de TOURVILLE sur ARQUES. De qui lui était venue cette petite seigneurie? Nous l'ignorons complètement.

Pierre DUCHESNE avait épousé Marie FOUACHE avant 1588 puisque d'après un acte du tabellionage de BACQUEVILLE leur fils Adrien avait 25 ans en 1613. Par suite de ce mariage, il allait devenir possesseur d'une autre fief le fief d'AUZOUVILLE sur la paroisse de SAINT OUEN PREND EN BOURSE ${ }^{3}$. Aujourd'hui le nom d'AUZOUVILLE y est totalement inconnu. Voici comment Pierre DUCHESNE devint propriétaire de ce fief.

Le ler Octobre 1603, après la mort de Salomon FOUACHE, écuyer, sieur d'AUZOUVILLE, eut lieu devant les tabellions de BACQUEVILLE le partage de ses biens entre ses frères et sœurs: lorsque selon la coutume de Caux, Antoine EUDES, Ecuyer, Seigneur de CATTEVILLE ${ }^{4}$ et de plusieurs autres fiefs et terres, époux de la fille cadette, présenta aux ainées les lots à choisir, Jean COQUEREL, écuyer, fils aîné de feue Marguerite FOUACHE eut pour sa part le manoir seigneurial d'AUZOUVILLE avec environ 88 acres de terre; Maître Pierre DUCHESNE au nom de sa femme Marie FOUACHE se fit attribuer le fief noble d'AUZOUVILLE avec une mazure et

1. Aujourd'hui le THIL-MANNEVILLE canton d'OFFRANVILLE.

2. Canton d'OFFRANVILLE.

3. St OUEN est aujourd'hui hameau de la commune de BERTREVILLE ST OUEN, Canton de LONGUEVILLE sur SCIE, à $13 \mathrm{Kms}$ de DIEPPE.

4. Paroisse de MANEHOUVILLE à $13 \mathrm{Kms}$ de DIEPPE. 
120 acres de terre; enfin Antoine EUDES eut pour lui le reste du domaine comprenant 80 acres environ.

On le voit par là, Pierre DUCHESNE ne manquait pas de biens et il était assez noblement apparenté. Nous insistons sur ce point afin de montrer que son petit fils Charles LE MOINE n'était pas du tout issu d'une famille vulgaire comme on l'a écrit, mais que par sa mère il tenait à la petite noblesse et à la bonne bourgeoisie.

Continuons maintenant notre généalogie:

Selon un acte du tabellionage de BACQUEVILLE en date du 18 Novembre 1626 Pierre DUCHESNE qui était alors décédé se qualifiait en son vivant avocat au Parlement. Le même acte nous apprend qu'Adrien était son fils aîné; puisque Charles LE MOINE était le neveu d'Adrien c'est que sa mère Judith DUCHESNE était la sœur d'Adrien. Nous avons done:

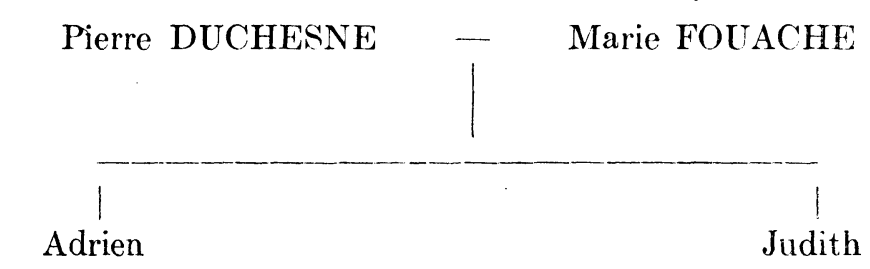

IIo) ADRIEN DUCHESNE :

Dès 1610 Adrien DUCHESNE prenait le titre de Sieur d'AUZOUVILLE ${ }^{1}$. Ce petit fief, nous l'avons vu, était entré dans la famille DUCHESNE par Marie FOUACHE. Adrien le reçut lors de son mariage ainsi qu'il le dit lui-même dans un acte de vente de 1622: je le possède, dit-il, " ...comme donataire de Maître Pierre DUCHESNE, Sieur d'IBERVILLE mon père et Marie FOUACHE ma mère par le traité de mariage d'entre ma femme et moi, de toute la succession, entant qu'il leur en peut appartenir, du feu Sieur d'AUZOUVILLE notre oncle " ${ }^{2}$.

En 1612 Adrien demeurait en la ville de DIEPPE dont il était bourgeois. Nous pouvons noter dès maintenant que si les DUCHESNE occupèrent une place notable dans la bourgeoisie de DIEPPE (un Pierre DUCHESNE en fut échevin et procureur syndic), il ne semble

1. T.B.-21 Décembre 1610.

2. T.B.-28 Septembre 1622. 
pas qu'ils aient été anoblis. Maintes fois les actes de tabellionage les qualifiaient de "noble homme ", mais dans le langage de l'époque cela signifie justement que s'ils exerçaient des fonctions réputées nobles, comme celle d'avocat, d'échevin etc... ils n'accédèrent pas à la noblesse; en effet ils ne se qualifièrent jamais d'écuyers, ce qui était le véritable titre de noblesse.

Bientôt, un acte du 19 Juïn $1613^{1}$ nous l'apprend, Adrien DUCHESNE vient habiter AUZOUVILLE. Voici cet acte: Noble homme Maitre Pierre DUCHESNE Sieur d'IBERVILLE et d'AUZOUVILLE, Damoiselle Marye FOUACHE sa femme et noble homme Adrien DUCHESNE, leur fils, atesté âgé, par les dits Sieurs et Damoiselle ces père et mère, de vingt cinq ans ou viron, le dit Adrien gouverneur des pages de la Rayne Marguerite, duchesse de Vallois demeurant assavoir le dit Sieur d'IBERVILLE et sadite femme à TOUSLESMESNILS, paroisse de LONGUEIL, et ledit Adrien DUCHESNE demeurant audit lieu d'AUZOUVILLE, paroisse de SAINT OUEN PREND EN BOURSE... "vendent à noble homme Maitre Jehan DELAPORTE conseiller du Roy et lieutenant en la Vicomté de ROUEN, demeurant à ROUEN paroisse SAINT PATRICE, quatre acres et demie de terre pour le prix de 675 Livres.

A partir de ce moment et pendant de nombreuses années encore, Adrien DUCHESNE sera domicilié à AUZOUVILLE. Etait-il réellement gouverneur des pages de la Reine Marguerite, première femme d'HENRI IV, répudiée en 1599 et décédée en 1615? Il est probable que c'était là un titre purement honorifique et peut-être acquis moyennant finances. Adrien DUCHESNE le portait encore quelques années après la mort de la Reine Marguerite.

Vivant en la paroisse de SAINT OUEN PREND EN BOURSE, Adrien DUCHESNE y faisait sans doute figure de gentilhomme campagnard: il surveillait, on peut le supposer, l'exploitation de ses terres et fréquentait les petits châtelains du voisinage; mais il ressort des actes de tabellionage que nous avons pu voir que ses affaires ne prospérèrent pas: il eut des procès, vendit des terres, même son fief d'AUZOUVILLE, il dut se retirer chez ses parents à TOUS LES MESNILS et finalement il disparut de la région.

1. T.B. 
De son mariage avec Suzanne LEDANOIS il eut au moins quatre enfants dont on trouve les actes de baptême dans les Registres de la paroisse de ST OUEN PREND EN BOURSE, déposés à la Mairie de BERTREVILLF SAINT OUEN.

Ce sont:

10 JEAN :baptisé le 10 Avril 1621. Il eut pour parrain Jean DELAPORTE Conseiller du Roi et son lieutenant en la Vicomté de ROUEN. La marraine fut Marie FOUACHE, grand-mère paternelle de l'enfant. Nous avons retrouvé la trace de ce Jean DUCHESNE dans les aveux rendus à la Seigneurie d'OMONVILLE dont la mouvance s'étendait jusqu'à AUZOUVILLE; il y est donné comme héritier sous bénéfice d'inventaire d'Adrien DUCHESNE son père; il fut avocat. Un autre Jean DUCHESNE qui semble bien être son fils fut bailly de Dieppe. 2o MAGDELEINE : baptisée le 2 Juin 1622 . Elle eût pour parrain Pierre EULDE, et pour marraine Marie LEGRAND sur lesquels nous n'avons aucun renseignement.

3o) MARIE : baptisée le 30 Mai 1623 . Elle eut pour parrain Charles LACAILLE, seigneur du Qilleul. Le Tilleul était un petit fief situé sur la paroisse de BACTUEVILLE et voisin d'AUZOUVILLE. La marraine fut damoiselle Marie LE POIGNEUX, femme de Jean de Mallet, écuyer, seigneur de SAINT OUEN, autre voisin de campagne.

4o) RADEGONDE: baptisée le 11 Octobre 1624. Elle eut pour parrain " Anthoine LEDANOIS " probablement un frère de sa mère. A cette époque vivait à SOTTEVILLE SUR MER un Jacques LE DANOIS, écuyer, sieur de la MOTHE qui était, c'est probable, de la même famille. La marraine fut Radegonde DELAPORTE : Avant son mariage avec Jean DELAPORTE elle s'appelait Radegonde de ROISSEY; c'était la sœur d'Urbain de ROISSEY, le célèbre corsaire, compagnon de Belain d'ESNAMBUC à l'ile de ST CHRISTOPHE en 1625; nous le savons par une procuration passée devant BEAUNEY tabellion à BACQUEVILLE, vers la même époque ${ }^{1}$.

Voici donc, en nous aidant des actes de baptême ci-dessus et de quelques aveux trouvés au chateau d'OMONVILLE, la descendance d'Adrien DUCHESNE :

1. Malheureusement le document est en si mauvais état que la date n'en est plus lisible. 


\section{ADRIEN DUCHESNE - SUZANNE LEDANOIS}
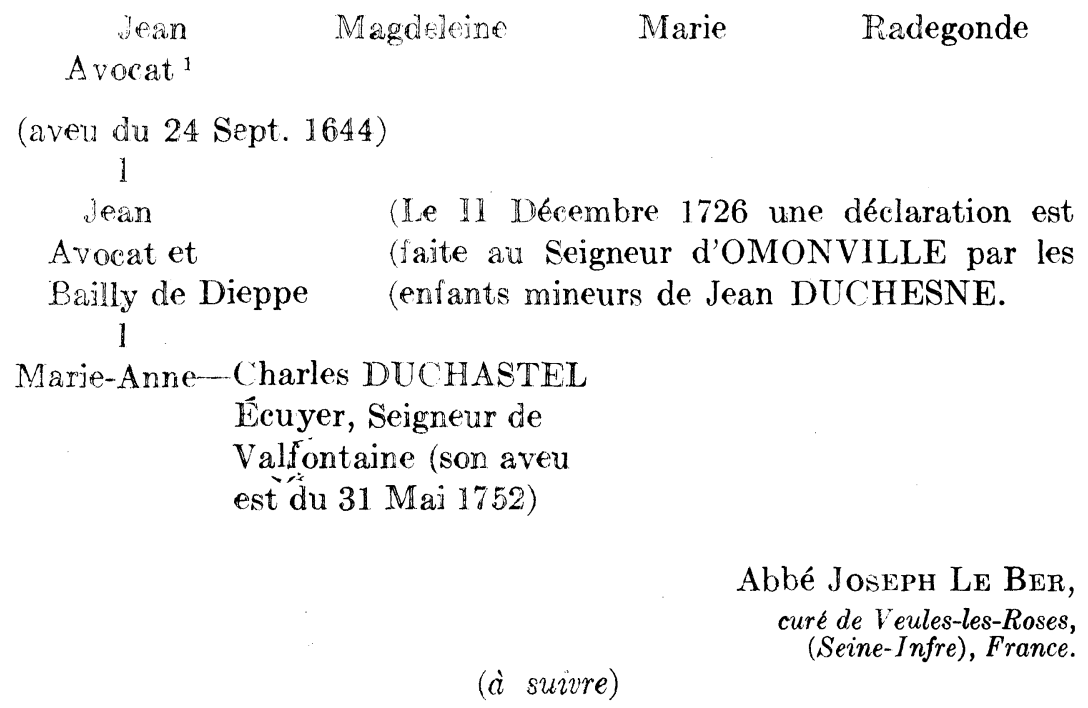

1. Cet aveu rendu par le fiss d'Adrien laisse supposer que celui-ci était décédé à cette date. 